\title{
UMA ANÁLISE DA CONSCIÊNCIA ECOLÓGICA PARA O CONSUMO "VERDE" NO VAREJO SUPERMERCADISTA
}

\author{
Sergio Silva Braga Júnior \\ Doutorando em Administração pela Universidade Nove de Julho - UNINOVE \\ sergio.bragajunior@gmail.com \\ Dirceu da Silva \\ Doutor em Educação pela Universidade de São Paulo - USP \\ Professor do Programa de Mestrado e Doutorado em Administração \\ Universidade Nove de Julho - PMDA/UNINOVE \\ dirceuds@uninove.br
}

\section{Sérgio Luiz do Amaral Moretti}

Doutor em Ciências Sociais pela Pontifícia Universidade Católica de São Paulo - PUC Professor do Programa de Mestrado e Doutorado em Administração - PMDA

Universidade Nove de Julho - UNINOVE sergiomoretti@uol.com.br

\section{Evandro Luiz Lopes}

Doutor em Administração de Empresas pela Universidade Nove de Julho - UNINOVE

Professor do Programa de Mestrado e Doutorado em Administração

Universidade Nove de Julho - PMDA/UNINOVE

\section{RESUMO}

elldijo@uol.com.br

A investigação sobre o envolvimento dos consumidores com produtos verdes vem tomando crescente importância. Se esse desenvolvimento passa por conhecer graus de consciência ecológica, voluntarismo e, também a adoção de um modo de vida simples por parte da sociedade, pretende-se, por meio deste artigo, verificar os principais fatores que influenciam consumidores a comprar no varejo supermercadista produtos considerados verdes. Para atingir o objetivo proposto, foi aplicado um survey junto a uma amostra de 837 respondentes, cujo instrumento de pesquisa foi trabalhado a partir da análise da literatura deste campo de estudos. No primeiro item da escala, buscou-se identificar a frequência de compras dos respondentes, com o propósito de retirar da amostra indivíduos que frequentam pouco ou raramente o varejo supermercadista. A análise dos resultados mostrou que as três hipóteses formuladas na pesquisa são suportadas pelo modelo calculado, pois apresentam valores dos coeficientes de caminhos ou cargas acima de 0,60 , indicando que são substanciais para sustentar ou suportar as hipóteses. A escala aplicada se revelou eficaz para testar a consciência ecológica e o consumo verde ou ambientalmente correto pelo consumidor.

Palavras-chave: Consciência ecológica; Consumo ambientalmente correto; Consumo verde; Supermercados; Varejo.

\section{AN ANALYSIS OF ECOLOGICAL AWARENESS OF "GREEN" CONSUMPTION ON RETAIL SUPERMARKET}

\section{ABSTRACT}

Cardigan (2008) stressed the importance of ecological awareness, volunteerism, and the adoption of a simple life to become more involved with green products. Thus, this study aims to determine the main factors that influence supermarket consumers to buy retail consumer products considered green. To reach that goal we conducted a survey with a sample of 837 respondents. With the first item of the scale, we sought to identify the frequency of respondents' purchases in order to exclude individuals who rarely or never shop at retail supermarkets. Thus, 604 responses were considered. The results showed that the three research hypotheses were supported by the calculated model, because their coefficients of paths or loads above 0.60 indicated that they are substantial to sustain or support the hypotheses. Thus, the scale proved effective when applied to test the ecological awareness against social and environmental rewards of companies, green consumption, and environmentally friendly consumption by the consumer.

Keywords: Green consumerism; Ecological consciousness; Retail.

* Apoio recebido do Fundo de Apoio a Pesquisa - FAP/UNINOVE 


\section{INTRODUÇÃO}

Produtos verdes são tidos como não prejudiciais ao meio ambiente e à saúde humana, tanto em seu conteúdo quanto em sua embalagem (Dashefsky, 1997; Jacobi, 2006; Ottman, 1994; Portilho, 2010). Sua viabilidade só se concretizou quando a discussão da questão ambiental se deslocou da produção para o consumo. Tal movimento se deve à emergência da preocupação que atingiu as empresas e o grande público a respeito do impacto causado pelo atual consumismo das sociedades industrializadas. Este fato viabilizou o lançamento de produtos verdes pela adesão das empresas a um mote sensível e de grande empatia para os consumidores (Elkington, Hailes, e Makower, 1990; Oliveira, Gouvêa, Gualhardi, 2004; Portilho, 2010). Esse processo pode ser visto como uma consequência do desenvolvimento da sociedade ocidental, à medida que diversos atores se conscientizam do espaço de discussão importante, interdisciplinar e de grande relevância para a sociedade e os consumidores (Karna, Hansen, Juslin, 2003; McCormick, 1992; May, Lustosa, Vinha, 2003; Urdan, 2001; Vogel, 2005).

A partir da Rio92 se iniciou um esforço, contínuo e crescente, de adesão por parte da comunidade empresarial (Fabris, 2008; Gomes e Moretti, 2007). Embora, possa ser visto como uma apropriação ideológica do movimento ecológico, já que era apontado como o seu maior causador (Moretti, 2010), seu esforço em situar o lado econômico do debate não poderia ser excluído do problema (Greenfield, 2004; Layrargues, 1998; Pringle e Thompson, 1999; Sheth e Parvatiyar, 1995). Nos últimos anos, procurando se adaptar a essas tendências, mais empresas estão incorporando ao seu cotidiano e ao planejamento estratégico práticas de sustentabilidade por meio de gestão ambiental e ações sociais junto às comunidades (Barbieri, 2004; Gomes e Moretti, 2007; Porter e Kramer, 2005). Na maioria das vezes, tais práticas são ligadas à gestão ambiental (Creyer e Ross, 1997; Miles e Covin, 2000; Seyfang e Paavola, 2008).

As empresas passaram a ter não somente controle sobre seus processos, mas, também uma gestão voltada para os aspectos socioambientais como propósito de fornecer suporte aos seus clientes e fornecedores (Braga, 2007; Parente, 2000; Puncheva, 2008). Conforme apontado por Moysés, Rodrigues e Moretti (2009), a gestão das ações socioambientais das empresas é fortemente ligada aos seus stakeholders, posto que sua reputação irá depender, em um grau elevado, da seriedade com que gerencia estas ações entre as partes. Na mesma linha, Donha (2002) expõe que o sucesso desta iniciativa está vinculado aos princípios básicos de sustentabilidade que devem ser conhecidos por todos os stakeholders: 1) a retirada de recursos de natureza não deve ir além de sua capacidade de reposição; 2) à natureza, não deve ser adicionado resíduos em quantidade acima de sua capacidade de absorção e, 3) para poder contribuir com o meio ambiente, é necessário a formação de uma relação harmônica, e não de domínio, sobre os recursos naturais.

Transcendendo a tradicional polarização exercida por produtores e consumidores, recentemente o varejo, situado em uma posição privilegiada na cadeia de venda de produtos ao consumidor final, foi alçado a uma posição na qual pode exercer alguns papéis que contribuam para a sustentabilidade (Parente e Gelman, 2006). O setor supermercadista acompanha esta evolução (Bedante e Slongo, 2004) demonstrada pelo fato de que lojas médias e grandes já oferecem grande variedade de produtos verdes (Braga, 2007; Parente, 2000). O aperfeiçoamento da eficiência organizacional e do relacionamento com os clientes (Tat Keh e Park, 1997), uma boa gestão de custos e preço de venda (Wilder, 2003) e, principalmente, maior cuidado com o descarte de produtos não utilizados (Braga, 2007) são fatores importantes para as empresas varejistas obterem sucesso neste novo contexto.

Este estudo procurou contribuir para o campo do consumo de produtos verdes, tendo como base uma pesquisa empírica que envolveu uma amostra de consumidores na cidade de São Paulo e lojas de varejo supermercadistas. Foi aplicado um questionário desenvolvido a partir da literatura analisada e tendo como objetivo verificar os principais fatores que influenciam os consumidores a comprar no varejo supermercadista produtos de consumo considerados verdes e sua relação com a 
sua consciência ecológica, ou seja, a percepção individual do que seja uma atitude ambientalmente correta.

O artigo está estruturado da seguinte forma: a seguir, é apresentada a revisão da literatura sobre o tema, os procedimentos metodológicos utilizados na pesquisa, uma discussão dos resultados e as considerações finais.

\section{FUNDAMENTAÇÃO TEÓRICA}

A literatura internacional, notadamente a norte-americana, tem se preocupado em verificar quais aspectos dos produtos verdes se diferenciam dos demais. Luchs, Naylor, Irwin e Raghunathan (2010) mostram que grandes empresas do varejo como Wal-Mart e Whole Foods têm se esmerado em lançamentos de produtos com o apelo verde e sustentável (Irwin e Naylor, 2009; Luthans e Youssef, 2007; Porges, 2007; Xueming e Battacharya, 2006). Por meio desses estudos, pode-se perceber que os consumidores não escolhem os produtos somente por seu apelo verde, mas, também pelo tipo de benefício que eles oferecem (Morh e Webb, 2005).

Esse fato torna os estudos, nesse campo, mais próximos das linhas tradicionais do comportamento do consumidor, à medida que tiram a carga excessiva do apelo ético dos referidos produtos. Não há evidências concretas de que os consumidores valorizam o apelo ético além das suas necessidades e quando não trazem benefícios para as empresas (Bamberg, 2003; Sem, Bhattacharya, 2001; Sisodia, Shet, Wolfe, 2007; Xueming e Bhattacharya, 2006). Simpson, Carmon, Dhar, Drolet e Nowlis (2001) mostraram que uma das decisivas mudanças surgidas no período foi a predominância dos aspectos cognitivos sobre os demais. Um fator decisivo para entender esse processo é aceitar que os indivíduos, muitas vezes, afirmam adotar valores que eles, de fato, não têm (Luchs et al., 2010). Tal cuidado deve ser adotado nos modelos de pesquisa para que não se tome as afirmações dos sujeitos por seu valor de face.

No Brasil, os estudos têm sido modestos. Pertence ao Instituto Ethos e o Instituto Akatu as iniciativas mais concretas, no sentido de entender o grau de consciência para o consumo consciente - na terminologia usada por eles (Gisi, 2008). Os dois institutos começaram uma série de pesquisas para descobrir o perfil deste tipo de consumidor em 2003 com a versão mais recente em 2010. A pesquisa gira em torno de uma segmentação de quatro tipos baseada no grau de adesão que os consumidores têm em relação a uma série de 13 itens. O fundamento desses itens se dá sobre os conceitos de atitude - grau de adesão aos valores e conceitos propostos e, comportamento - ações ligadas à prática cotidiana dos preceitos ambientais e de responsabilidade social (Instituto Akatu, 2010, p. 7). A classificação dos segmentos é baseada no critério: apresentar sempre um determinado número de comportamentos e são indiferentes; com a adesão de dois comportamentos; iniciantes, de três a sete comportamentos; engajados, de oito a dez comportamentos; e, conscientes, de onze a treze comportamentos (Instituto Akatu, 2010). Os resultados da última edição mostram um crescimento dos consumidores indiferentes e a estabilização dos consumidores conscientes. Independentemente dos resultados, o importante é que o modelo não tem sido muito utilizado pelos pesquisadores nacionais que só recentemente começaram a se interessar pelo tema e preferiram conduzir suas próprias pesquisas.

Motta e Rossi (2001) estão entre os primeiros a estudar a relação entre fator ecológico e, consumo no Brasil. A pesquisa mostrou que o meio ambiente, no momento do estudo, ainda não era considerado uma variável importante na tomada de decisão pelos consumidores. Contudo, os autores sugerem que este fator, presente nas considerações da amostra, poderia se tornar importante no futuro e propuseram à comunidade empresarial que a levasse em consideração em sua estratégia de longo prazo.

Bertollini e Possamai (2005) propuseram um instrumento de pesquisa para medir o grau de consciência ambiental dos consumidores e os critérios utilizados no momento da compra. Seu instrumento baseado em estudos preliminares de consumo consciente (Maia e Vieira, 2004; Raposo, n.d.), contudo não fornecia elementos além da consciência ecológica, não permitindo confrontar 
esta condição com a ação de compra. Da mesma forma, ainda preliminarmente, Pato e Tamayo (2006) fizeram um estudo exploratório com universitários sobre o comportamento ecológico de indivíduos, sem relacioná-lo ao comportamento de compra. Com base nas escalas de Kaiser (1998) e Karp (1996) eles desenvolveram a Escala de Comportamento Ecológico (ECE) que foi testada e adaptada à realidade brasileira. Os resultados indicaram que o comportamento ecológico tem aspectos multidimensionais, com predominância dos fatores: ativismo, consumo, economia de água e energia, limpeza urbana e reciclagem.

Aspectos, tais como a atitude, percepção do consumidor e instrumentos de avaliação são estudados e analisados para serem relacionados a consciência ecológica (Bedante e Slongo, 2004) ou para buscar evidências de um consumo que seja focado na redução de impactos ambientais e sociais. Cardigo (2008) ressaltou a importância da consciência ecológica, do voluntarismo e da adoção de um modo de vida simples para um maior envolvimento com produtos verdes. Malheiros, Farhangmehr e Soares (2010) investigaram o gap percebido entre as atitudes, intenções e o comportamento, no que tange ao consumo ético.

A mensuração do grau em que os consumidores podem ser classificados como socialmente responsáveis e sua influência nas compras tem nos estudos brasileiros a ênfase voltada principalmente, para a linha proposta por Roberts (1996) cujo propósito é de alinhar as condutas declaradas dos consumidores com suas preferências no momento da compra. $\mathrm{O}$ autor propõe a Escala de Comportamentos Socialmente Responsáveis do Consumidor (ECSRC), com 18 sentenças que visam avaliar se há predomínio das preocupações sociais nas decisões de compra.

A ECSRC foi testada e validada no Brasil por Queiroga, Gouveia, Coutinho, Vasconcelos e Jesus (2005) por meio de uma amostra de universitários, cujas características, sem dúvida, ressaltam as limitações do estudo. Contudo, no que tange à adaptação da escala para a língua portuguesa, o estudo pode ser considerado exitoso.

Lages e Vargas (2002) replicaram um estudo baseado em uma pesquisa mais recente de Straughan e Roberts (1999), que consiste em uma espécie de desdobramento da pesquisa de Roberts (1996) que havia verificado a correlação de variáveis demográficas e psicográficas do consumidor e sua consciência ecológica que receberam novas variáveis para medir essas características. Os resultados mostraram uma baixa relação entre as variáveis comportamentais e as demográficas, entretanto as variáveis de relação entre consciência ecológica e comportamento de compra apresentaram uma variância explicada de $62,35 \%$, bastante alta se comparada com o estudo do qual se replicou a escala (Straughan e Roberts, 1999).

Atualmente, tais fatores vêm ganhando espaço no processo de busca de informação e avaliação de alternativas no Brasil. Algumas atitudes, tais como a percepção e o comportamento responsável do consumidor foram estudados por Bedante e Slongo (2004), Ceschim e Marchetti (2009), Garcia, Silva, Pereira, Rossi e Minciotti (2008), Leite e Santos (2007) e Seyfang e Paavola (2008) que conceituaram o consumo sustentável ou consciente como aquele focado na redução de impactos ambientais e sociais realizados, também pelo varejo. Ceschim e Marchetti (2009), por meio de discussões em grupo, mostraram a influência das características psicológicas e da inovação percebida em produtos orgânicos. Os resultados mostraram que os consumidores estão atentos quanto ao tipo de alimentação que consomem e que são sensíveis às questões de bem-estar.

Pelo exposto até o momento, e pela validação das escalas de Lages e Vargas (2002) para a consciência ecológica e sua relação com a compra de produtos ecologicamente corretos, é possível propor as seguintes hipóteses:

\section{H1 . Consciência ecológica influencia na compra de produtos verdes}

Garcia et al. (2008) desenvolveram uma escala própria e a validaram mostrando a relação entre a consciência ecológica e o comportamento de compra ambientalmente correta que está relacionada ao esforço das empresas em adotar uma abordagem ambiental em seus produtos e a recompensa recebida do consumidor prestigiando tal iniciativa. Em que pesem as limitações do 
estudo no quesito amostra de universitários, os procedimentos para o desenvolvimento da escala testada e o uso de equação estrutural para testar os construtos garantiu a validade do instrumento para estudos futuros.

Moretti, Silva e Braga (2010), usando a escala de Garcia et al. (2008), fizeram uma análise fatorial exploratória da qual emergiram dois construtos derivados do construto daquela escala: Comportamento ambientalmente correto, que ficou ligado aos aspectos de reconhecimento de produtos com menos embalagens e que agridem menos ou não agridem o meio ambiente (Garcia et. al, 2008), e compra verde, que ficou ligado a produtos orgânicos, livres de agrotóxicos e sem modificações (Lages e Vargas Neto, 2002). Baseado nesses autores, pode-se propor as seguintes hipóteses para esse estudo:

H2. Consciência ecológica influencia na compra de produtos ambientalmente corretos H3: Consciência ecológica gera recompensa às empresas ambientalmente corretas

Resumidamente, as hipóteses do estudo ficam como na Figura 1:

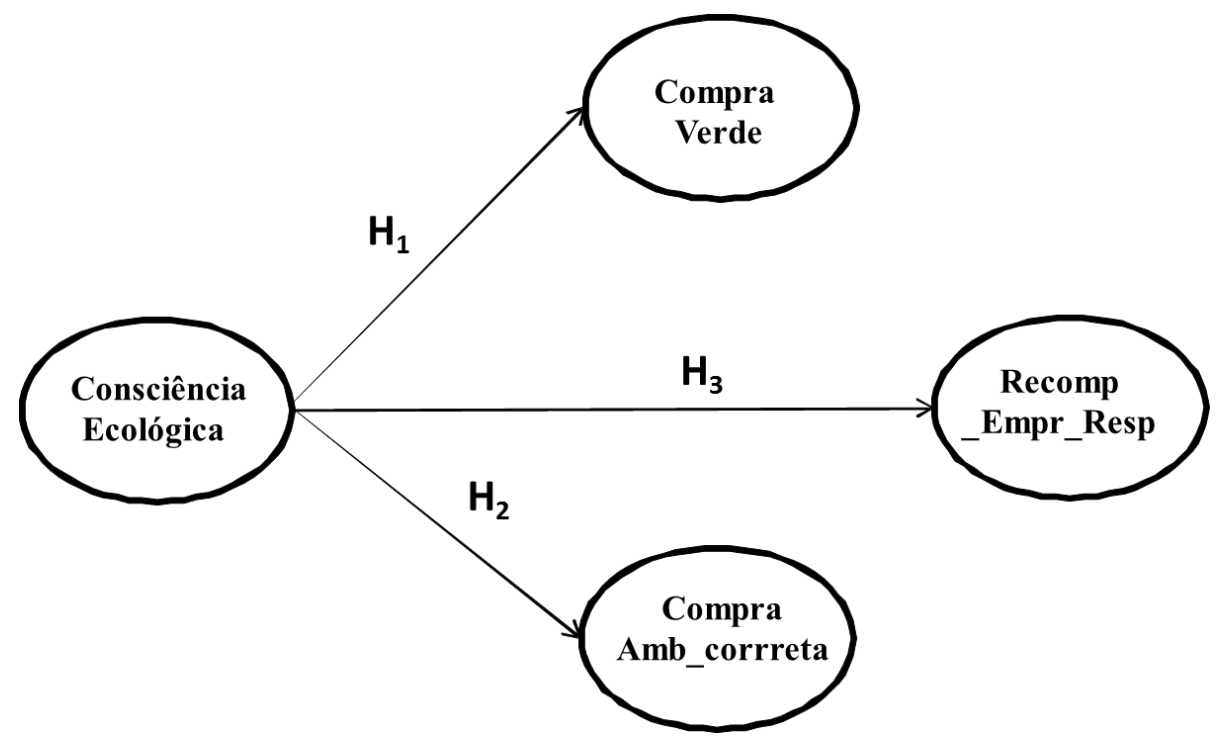

Figura 1: Modelo da pesquisa com as Hipóteses

Fonte: Elaborado pelos autores

\section{PROCEDIMENTOS METODOLÓGICOS}

Para atingir o objetivo proposto de verificar a consciência ecológica como fator de influencia para compra verde, para compra ambientalmente correta e para recompensar empresas ambientalmente corretas, foi aplicado um survey em uma amostra de 837 respondentes coletados na cidade de São Paulo e lojas de varejo supermercadistas. Os dados foram coletados entre os meses de outubro/2010 e janeiro 2011 e no primeiro item da escala, buscou-se identificar a frequência de compras dos respondentes com o propósito de retirar da amostra indivíduos que frequentam pouco ou raramente o varejo supermercadista. Assim, foram consideradas 604 respostas.

Como já exposto na revisão da literatura, o desenvolvimento da escala aplicada foi baseada em escalas já validadas, anteriormente, que procuraram analisar a consciência ecológica (Lages, Vargas, 2002) e a recompensa socioambiental responsável das empresas pelo consumidor (Garcia et al., 2008). A partir delas, foram selecionados os itens ou assertivas que refletiam o propósito do estudo. Seguindo a recomendação de Devillis (2003), a proposta das escalas que compõem o instrumento de pesquisa foi enviada para a análise de três especialistas escolhidos por sua reputação 
e conhecimento do tema. Tais especialistas avaliaram os itens da escala e validaram o modelo causal baseado na lógica das duas escalas (vide figura 1).

Por fim, a escala ficou estruturada em 24 itens ou assertivas (vide Quadro 1), cuja avaliação consistiu em uma escala do tipo Likert com cinco pontos de concordância/discordância. As variáveis categóricas da pesquisa foram definidas como sendo sexo, faixa de idade, faixa de renda familiar e estado civil. Para análise dos dados adotou-se o software SPSS 15.0, para os testes de freqüência, e o software SmartPLS 2.0 - M3 (Ringle, Wende, Will, 2005), para a avaliação da Modelagem de Equações Estruturais (MEE).

Como método principal de análise dos dados, a MEE foi eleita pelo motivo de esse método permitir a avaliação das relações causais entre construtos e o subsequente teste de hipóteses pela avaliação dos coeficientes de caminho (path coefficients).

Também foi usado o modelo de medidas Partial Least Square -Path Modeling(PLS-PM) ou Mínimos Quadrados Parciais - Modelagem de Caminhos, pois ao ser realizado o teste PK de Mardia (Jöreskog, Söbom, 1993) de aderência à uma distribuição normal multivariada, este se mostrou significante $(\mathrm{p}<0,001)$ e, portanto, os dados da matriz das variáveis medidas não respeitaram a distribuição multivariada desejada.

Os modelos de medidas para o cálculo da MEE foram aqueles que têm pressupostos de Distribuição Assintótica Livre, isto é, a não necessidade da normalidade multivariada. Mais especificamente três modelos poderiam ser usados: Diagonizaded Weighted Least Square(DWLS) ou Mínimos Quadrados Ponderados Diagonalizados, Weighted Least Square (WLS) ou Mínimos Quadrados Ponderados e PLS-PM. (Hair, Anderson, Tatham, e Black, 2009; Jöreskog, Söbom, 1993). Os dois primeiros necessitam de amostras muito extensas, pelo menos o dobro da obtida nesta pesquisa, e, assim, foram descartados, restando o PLS-PM como possibilidade adequada para a análise dos dados. O PLS-PM é uma alternativa com qualidade comprovada pela comunidade internacional de pesquisadores em vários campos do saber (Ringle et al., 2005) e permite uma maior plasticidade na análise dos dados. 


\begin{tabular}{|c|c|c|}
\hline Constructo & VARIÁVEIS DA ESCALA & FONTES \\
\hline Rec_Empr_Resp & $\begin{array}{l}\text { 1.Eu não compro produtos fabricados ou vendidos por empresas } \\
\text { que prejudicam ou desrespeitam o meio ambiente. }\end{array}$ & \multirow{3}{*}{ GARCIA et al., 2008} \\
\hline Rec_Empr_Resp & $\begin{array}{l}\text { 2.Eu não compro produtos para minha casa que prejudicamo } \\
\text { meioambiente. }\end{array}$ & \\
\hline Rec_Empr_Resp & $\begin{array}{l}\text { 3.Quando eu tenho que escolher entre dois produtos iguais, eu } \\
\text { sempre escolho o que é menos prejudicial às outras pessoas e ao } \\
\text { meio ambiente }\end{array}$ & \\
\hline Comp_Amb_Corr & $\begin{array}{l}\text { 4.Eu já convenci amigos ou parentes a não comprar produtos que } \\
\text { prejudicam o meio ambiente. }\end{array}$ & \multirow{5}{*}{$\begin{array}{l}\text { GARCIA et al., } 2008 \text { e } \\
\text { MORETTI et al., } 2010\end{array}$} \\
\hline Comp_Amb_Corr & $\begin{array}{l}\text { 5.Eu sempre faço um esforço para reduzir o uso de produtos feitos } \\
\text { de recursos naturais escassos. }\end{array}$ & \\
\hline Comp_Amb_Corr & $\begin{array}{l}\text { 6.Quando possível, eu sempre escolho produtos que causam } \\
\text { menor poluição. }\end{array}$ & \\
\hline Comp_Amb_Corr & 7.Eu procuro comprar produtos com menos embalagem possível & \\
\hline Comp_Amb_Corr & $\begin{array}{l}\text { 8.Eu evito comprar produtos com embalagens que não são } \\
\text { biodegradáveis. }\end{array}$ & \\
\hline Comp_Verde & 9.Eu compro produtos orgânicos porque são mais saudáveis. & \multirow{3}{*}{$\begin{array}{l}\text { LAGES \& VARGAS } \\
\text { NETO, } 2002\end{array}$} \\
\hline Comp_Verde & $\begin{array}{l}\text { 10.Eu prefiro alimentos sem agrotóxicos porque eles respeitam o } \\
\text { meio ambiente. }\end{array}$ & \\
\hline Comp_Verde & $\begin{array}{l}\text { 11.Eu estou disposto a pagar um pouco mais por produtos e } \\
\text { alimentos que estão livres de elementos químicos e que prejudicam } \\
\text { o meio ambiente. }\end{array}$ & \\
\hline Cons_Eco & $\begin{array}{l}\text { 12.Quando eu compro produtos e alimentos, as preocupações com } \\
\text { o meio ambiente interferem na minha decisão de compra. }\end{array}$ & $\begin{array}{l}\text { GARCIA et al., } 2008 \mathrm{e} \\
\text { MORETTI et al., } 2010\end{array}$ \\
\hline Rec_Empr_Resp & $\begin{array}{l}\text { 13.Eu não compro produtos e alimentos que podem causar a } \\
\text { extinção de algumas espécies animais e vegetais. }\end{array}$ & GARCIA et al., 2008 \\
\hline Cons_Eco & $\begin{array}{l}\text { 14.Eu compro produtos com certificação ambiental porque são } \\
\text { ambientalmente corretos. }\end{array}$ & \multirow{2}{*}{$\begin{array}{l}\text { GARCIA et al., } 2008 \text { e } \\
\text { MORETTI et al., } 2010\end{array}$} \\
\hline Cons_Eco & 15.Eu já troquei ou deixei de usar produtos por razões ecológicas. & \\
\hline Comp_Verde & $\begin{array}{l}\text { 16.A diferença de preço nem sempre é suficiente para privilegiar a } \\
\text { empresa ecologicamente correta. }\end{array}$ & \multirow{3}{*}{$\begin{array}{l}\text { LA GES \& VARGAS } \\
\text { NETO, } 2002\end{array}$} \\
\hline Comp_Verde & $\begin{array}{l}\text { 17.Pagaria mais para comprar produtos que promovam a proteção } \\
\text { ambiental. }\end{array}$ & \\
\hline Comp_Verde & 18.Pagaria mais para comprar produtos orgânicos. & \\
\hline Cons_Eco & $\begin{array}{l}\text { 19.Ando mais para comprar produtos que tenham uma certificação } \\
\text { ambiental. }\end{array}$ & \multirow{5}{*}{$\begin{array}{l}\text { GARCIA et al., } 2008 \mathrm{e} \\
\text { MORETTI et al., } 2010\end{array}$} \\
\hline Comp_Amb_Corr & $\begin{array}{l}\text { 20.Na compra não é importante que a empresa seja ambientalmente } \\
\text { responsável. }\end{array}$ & \\
\hline Cons_Eco & $\begin{array}{l}\text { 21.Busco sempre procurar informações sobre as certificações } \\
\text { ambientais dos fabricantes de produtos que eu compro. }\end{array}$ & \\
\hline Cons_Eco & $\begin{array}{l}\text { 22.Presto atenção nos produtos destacados nas gondulas como } \\
\text { ambientalmente corretos }\end{array}$ & \\
\hline Cons_Eco & $\begin{array}{l}\text { 23.As seções de produtos orgânicos e ambientalmente corretos } \\
\text { são facilmente identificadas por mim }\end{array}$ & \\
\hline
\end{tabular}

Quadro 1: Escala usada na pesquisa, indicando a relação com os seus respectivos construtos e com as fontes que foram usadas para os seus itens

Fonte: Elaborado pelos autores 


\section{ANÁLISE DOS DADOS}

Para se descrever as características das amostras, foram realizadas análises de frequências. Obteve-se que $50.7 \%$ eram respondentes do sexo feminino e $49.3 \%$, do sexo masculino; $44.3 \%$ eram solteiros e $55.7 \%$, casados; $3 \%$ com idades até 20 anos, $32.6 \%$, de 21 a 30 anos, $28.5 \%$ com idades de 31 a 40 anos, $25.3 \%$, de 41 a 50 anos e $10.6 \%$, acima de 50 anos, indicando uma amostra equilibrada com respeito ao sexo e estado civil e bem distribuída nas faixas de idades de consumidores entre 21 e 50 anos.

Com base nos dados obtidos pela pesquisa, foi estimado um modelo reflexivo, no qual os indicadores são as manifestações do construto. A consciência ecológica ficou como influenciadora para os outros construtos, conforme as hipóteses estipuladas pela pesquisa (vide figura 1).

$\mathrm{Na}$ análise da validade do modelo, foi gerada a carga fatorial dos itens dentro de cada construto e seus respectivos $\mathrm{R}^{2}$, conforme mostra a Figura 2.

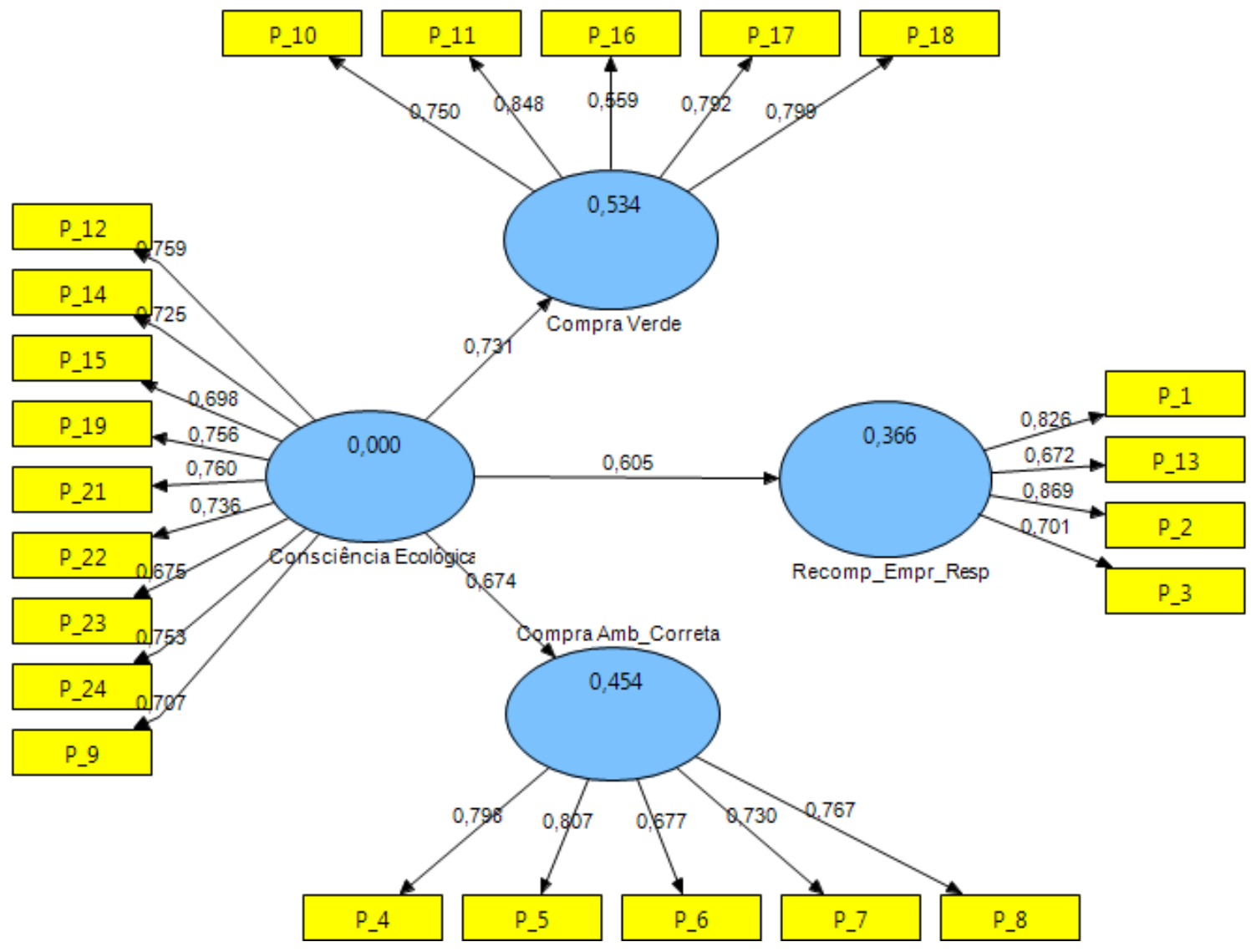

Figura 2: Modelo ajustado na pesquisa pelo uso da MEE PLS-PM

Fonte: Elaborado pelos autores

Nota: Todos os coeficientes estruturais se mostraram significantes $(\mathrm{p}<0,001)$. Significância estimada por meio do método de reamostragem (bootstrap) com 1000 repetições (Ringle et al., 2005)

A variável P_20 foi retirada do modelo, pelo motivo de apresentar carga fatorial menor de 0,50, indicado ajuste de baixa qualidade ao modelo geral. Uma análise simples por meio de tabulação cruzada, com as variáveis $P_{-}$2, escolhida por apresentar maior carga fatorial no modelo, mostrou que não há coerência nas respostas dos sujeitos. Talvez, pela análise das semânticas das variáveis (20. Na compra não é importante que a empresa seja ambientalmente responsável) não houve uma compreensão dos seus propósitos, pois é difícil avaliar o compromisso da empresa com 
o meio ambiente. Em seguida, foram avaliadas as variâncias médias extraídas (AVE), confiabilidade composta, $\mathrm{R}^{2}$ e Alfa de Cronbach(1996) dos construtos (Tabela 1).

Para avaliação do modelo estrutural, o critério essencial é o coeficiente de determinação $\left(\mathrm{R}^{2}\right)$. Coeficientes de determinação até 0,19 são considerados fracos; de 0,33 até 0,66 , moderados e acima de 0,67, substanciais, de acordo com Henseler, Ringle, Sinkovics, 2009. Avaliado os $\mathrm{R}^{2}$, constata-se que são considerados moderados. Ainda, os valores das AVEs estão acima daquele entendidos como referenciais $(>0,50)$, além dos valores da Confiabilidade Composta e dos Alfas de Cronbach, indicando que o modelo está bem ajustado e apresenta qualidade para ser interpretado.

Tabela 1: Critérios de qualidade dos ajustes do modelo - Especificação do MEE Valores da variância média extraída (AVE), confiabilidade composta, $\mathrm{R}^{2} \mathrm{e}$ Alfa de Cronbach dos Contrutos

\begin{tabular}{|l|c|c|c|c|}
\hline \multicolumn{1}{|c|}{ Constructo } & AVE & Confiabiliade Composta & R quadrado & Alfa de Cronbach \\
\hline Consciência Ecológica & 0,534 & 0,911 & $* * *$ & 0,890 \\
\hline Compra Amb_Correta & 0,572 & 0,870 & 0,454 & 0,808 \\
\hline Compra Verde & 0,572 & 0,868 & 0,534 & 0,890 \\
\hline Recomp_Empr_Resp & 0,595 & 0,853 & 0,366 & 0,768 \\
\hline Valores Refernciais & $\mathbf{> 0 , 5 0}$ & $>0,70$ & vide texto & $>\mathbf{0 , 6 0}$ \\
\hline
\end{tabular}

Fonte: Elaborado pelos autores

Dando sequência, foi analisada a raiz quadrada das AVEs (RMS-AVE) de cada construto com seus coeficientes de correlação de Pearson para confirmar a validade discriminante dos dados e que são apresentados na Tabela 2. O modelo apresenta validade discriminante quando as RMSAVE são maiores que os respectivos coeficientes de correlação de Pearson. Observa-se que, na Tabela 2, os valores da RMS-AVE são maiores que as correlações, indicando que há validade discriminante do modelo.

Tabela 2: Comparação dos quadros das AVEs versus correlação dos construtos (em cinza)

\begin{tabular}{|l|c|c|c|c|}
\hline & $\begin{array}{c}\text { Compra } \\
\text { Amb_Correta }\end{array}$ & $\begin{array}{c}\text { Compra } \\
\text { Verde }\end{array}$ & $\begin{array}{c}\text { Consciência } \\
\text { Ambiental }\end{array}$ & $\begin{array}{c}\text { Recomp_Empr_ } \\
\text { Resp }\end{array}$ \\
\hline $\begin{array}{l}\text { Compra } \\
\text { Amb_Correta }\end{array}$ & $\mathbf{0 , 7 5 7}$ & & & \\
\hline Compra Verde & 0,570 & $\mathbf{0 , 7 5 6}$ & & \\
\hline $\begin{array}{l}\text { Consciência } \\
\text { Ecológica }\end{array}$ & 0,673 & 0,730 & $\mathbf{0 , 7 3 0}$ & \\
\hline $\begin{array}{l}\text { Recomp_Empr_- } \\
\text { Resp }\end{array}$ & 0,582 & 0,534 & 0,559 & $\mathbf{0 , 8 3 7}$ \\
\hline
\end{tabular}

Fonte: Elaborado pelos autores

Ainda, para se avaliar a qualidade geral do modelo, calculou o indicador Goodness-of-Fit (GoF) que é dado pela média geométrica do $\mathrm{R}^{2}$ médio e a AVE média (Tenenhuaus et al., 2005, citado em Bido, Godoy, Ferreira, Moreira, e Scartezini, 2011). O valor calculado foi de 0,512, o que indica que o modelo foi bem ajustado, pois, segundo os autores, valores acima de 0,36 são considerados bons para áreas como as Ciências Sociais.

Uma vez confirmada a qualidade dos ajustes do modelo, pode-se fazer inferências sobre os coeficientes de caminho e sobre os seus valores, já que estando o modelo ajustado, esses valores podem ser usados para se avaliar as hipóteses advindas da teoria (vide Tabela 3 ) 
Tabela 3: Avaliação das hipóteses apresentadas na pesquisa

\begin{tabular}{|c|l|c|c|}
\hline Hipótese & \multicolumn{1}{|c|}{ Caminho } & Carga & Conclusão \\
\hline $\mathbf{H 1}$ & Consciência Ecológica ==> Compra Verde & 0,731 & Suportada \\
\hline $\mathbf{H 2}$ & $\begin{array}{l}\text { Consciência Ecológica ==> Compra } \\
\text { Ambientalmente Correta }\end{array}$ & 0,674 & Suportada \\
\hline $\mathbf{H 3}$ & $\begin{array}{l}\text { Consciência Ecológica }==>\text { Recompensa as } \\
\text { Empresas Ambientalmente Corretas }\end{array}$ & 0,605 & Suportada \\
\hline
\end{tabular}

Fonte: Elaborado pelos autores

\section{CONSIDERAÇÕES FINAIS}

Este estudo procurou contribuir para o campo do consumo de produtos verdes a partir de uma pesquisa que envolveu um survey aplicado em uma amostra de 604 respondentes coletados na cidade de São Paulo e de lojas de varejo supermercadistas. Foi aplicado um questionário desenvolvido com base na literatura analisada e tendo como objetivo verificar os principais fatores que influenciam os consumidores a comprar no varejo supermercadista produtos de consumo considerados verdes e sua relação com sua consciência ecológica, ou seja, a percepção individual do que seja uma atitude ambientalmente correta.

A análise da Tabela 3 acima mostra que as três hipóteses formuladas na pesquisa são suportadas pelo modelo calculado, pois apresentam valores dos coeficientes de caminhos ou cargas acima de 0,60 . Tais valores podem ser interpretados como substanciais para sustentar ou suportar as hipóteses. As escalas aplicadas se revelaram eficazes para testar a consciência ecológica (Lages, Vargas, 2002) e a recompensa socioambiental responsável das empresas pelo consumidor (Garcia et al., 2008).

Pode-se dizer, com base nos resultados, que a consciência ecológica, ou seja, a percepção de que há uma conduta ambientalmente correta a ser seguida por pessoas e empresas, é um importante influenciador no processo de decisão de compra de produtos, sejam verdes ou ambientalmente corretos. As teorias analisadas preveem a relação entre consciência ambiental ecológica (Lages \&Vargas, 2002) e atitude no momento da compra (Bedante e Slongo, 2004) ou entre consciência ambiental e comportamento ambientalmente correto e sua influência na decisão de compra (Garcia et al., 2008; Leite e Santos, 2007). Os resultados mostraram que este situação parece ser correta e deve continuar sendo explorada, com o desenvolvimento de novas escalas que procurem outras relações como, grau de instrução, postura individual versus familiar e ou do grupo de referência.

Foi possível observar que existe uma grande preocupação quanto aos aspectos ambientais e sobre o que a sociedade deve fazer para reduzir o impacto ambiental, mas, por outro lado, foi notório o comportamento do consumidor em não recompensar, de forma clara e substancial, as ações das empresas, pois, na hipótese $\mathrm{H} 2$, o $\mathrm{R}^{2}$ ficou em 0,366 , sendo considerado um valor limite para classificação como moderado, podendo-se dizer que o consumidor está começando a perceber as ações socioambientais realizadas pelas empresas e passando a recompensá-las (Henseler et al., 2009).

Quanto à compra verde (H1), onde os itens que estão compondo o constructo remetem ao comportamento de compra de produtos orgânicos, livres de agrotóxicos e sem modificações, a influência da consciência ecológica é maior em relação a hipótese $\mathrm{H} 2(0,534)$. Assim, pode-se entender que a atenção a qualidade de vida e dos produtos (que representam um comportamento mais saudável) tem uma relação maior com a consciência ecológica do que a preocupação com a geração de resíduos no meio ambiente medida pela compra ambientalmente correta.

Isto é demonstrado quando a compra ambientalmente correta (H3) é medida e, a aceitação do consumidor também se apresenta moderada, permitindo aos pesquisadores acreditar que existe uma tendência de aceitação ao estilo de compra verde que está ligada a produtos que geram menos impacto ambiental, tanto em sua composição quanto em sua apresentação (embalagem), conforme 
itens que compõem o constructo. Desta forma, é possível inferir que a aceitação do consumidor para este tipo de produto pode estar aumentando.

Há que se reconhecer a limitação do estudo atual que trabalhou com uma amostra por conveniência e bastante homogênea, embora equilibrada em termos de gênero e idade, mas devido a essa restrição não se pode ir além da constatação destes resultados para a própria população estudada. Por outro lado, os resultados do estudo permitem avançar um pouco mais o conhecimento sobre o tema, sendo possível observar que consciência ecológica existe, mas que ainda não está refletindo, de forma substancial, no comportamento de compra e na recompensa as empresas ambientalmente corretas.

A contribuição deste estudo para a academia foi ampliar um pouco mais o campo de investigação sobre a avaliação pelos consumidores dos produtos verdes. Muito se fala neste campo, mas poucas são as evidências de sucesso e fracassos destas iniciativas. Os esforços feitos pelos pesquisadores são pontuais e localizados. De fato, foi mostrado na literatura que o interesse sobre o assunto é grande em todos os setores da sociedade e a mobilização das empresas em prol de serem reconhecidas como boas praticantes da sustentabilidade é um sinal evidente. Contudo, ainda não há um consenso estabelecido ou mesmo estudos que esgotem o tema de forma sistemática.

Da mesma forma, a contribuição para a área gerencial se dá na mesma direção, porém devese ressaltar que a aproximação entre a academia e o mercado ainda deixa a desejar, com pouca troca de informações, por exemplo, das pesquisas realizadas pelas empresas para sustentar o desenvolvimento e lançamento de produtos verdes. Neste aspecto, pouco se sabe e muito menos são divulgados nos congressos e seminários acadêmicos. Por isso, é necessário mais esforço para ligar estas duas importantes fontes de informação sobre o tema. Uma perspectiva de continuidade para este estudo se dá, exatamente no sentido de investigar produtos que já estão disponíveis no mercado, por meio da comparação dos aspectos internos, como as pesquisas, que sustentaram seu desenvolvimento e lançamento e sua avaliação atual pelos consumidores.

\section{REFERÊNCIAS}

Bamberg, S. (2003). How does environmental concern influence specific environmentally related behaviors? A new answer to an old question. Journal of Environmental Psychology, 23, 21-32.

Barbieri, J. C. (2004). Gestão socioambiental empresarial: conceitos, modelos e instrumentos. São Paulo: Saraiva.

Bedante, G. N., Slongo, L. A. (2004). O comportamento de consumo sustentável e suas relações com a consciência ambiental e a intenção de compra de produtos ecologicamente embalados. Anais do Encontro de Marketing, Porto Alegre, RS, Brasil, 1.

Bertollini, G. R. F., Possamai, O. (2005). Proposta de instrumento de mensuração do grau de consciência ecológica ambiental, do consumo ecológico e dos critérios de compra dos consumidores. Revista de Ciência e Tecnologia, 13(25/26) 17-25.

Bido, D. S., Godoy, A. S., Ferreira, J. F., Moreira, J., e Scartezini, V. N. (2011). Examinando a relação entre aprendizagem individual, grupal e organizacional em uma instituição financeira. Revista Eletrônica de Administração, 17(1), 58-85.

Braga, S. S., Jr. (2007). Gestão ambiental no varejo: um estudo das práticas de logística reversa em supermercados de médio porte. Dissertação de Mestrado, Faculdade de Economia, Administração e Contabilidade, Ribeirão Preto, SP, Brasil. 
Cardigo, A. C. J. (2008). The adoption of ecological conscious consumer behavior: exploring the association with materialism and voluntary simplicity lifestyles. Master Dissertation at ISCTE Business School, Lisboa, PT.

Ceschim, G., Marchetti, R. Z. (2009). O comportamento inovador entre consumidores de produtos orgânicos: uma abordagem qualitativa. Anais do Encontro da Associação Nacional de PósGraduação e Pesquisa em Administração, São Paulo, SP, Brasil, 33.

Creyer, E. H., Ross, W. T., Jr. (1997). The influence of firm behavior on purchase intention: do consumers really care about business ethics? Journal of Consumer Marketing, 14(6), 421-432.

Cronbach, L. J. (1996). Fundamentos da testagem psicológica (5a ed.). Porto Alegre: Artes Médicas.

Dashefsky, S. (1997). Dicionário de ciência ambiental: guia de A a Z. São Paulo: Gaia.

Devillis, R. F. (2003). Scale development: theory and applications. Thousand Oaks: Sage Publications.

Donha, M. S.(2002). Conhecimento e participação da comunidade no sistema de gerenciamento de resíduos sólidos urbanos: o caso de Marechal Cândido Rondon - PR. Dissertação de Mestrado, Universidade Federal de Santa Catarina, Florianópolis, SC, Brasil.

Elkington, J., Hailes, J., \& Makower, J. (1990). The green consumer. New York: Penguin.

Fabris, C. (2008). Marketing verde nos sites das maiores empresas do país: um estudo com fundamento na 'legitimidade' e 'isomorfismo'. Anais do Encontro de Marketing, Curitiba, PR, Brasil, 3.

Garcia, M. N., Silva, D., Pereira, R. S., Rossi, B. G. e Minciotti, S.A. (2008). Inovação no comportamento do consumidor: recompensa às empresas socioambientalmente responsáveis. Revista de Administração e Inovação, 5(2), 73-91.

Gisi, A. C. (2008). Análise do comportamento de consumo sob o enfoque da sustentabilidade estudo de caso: município de Chapecó-SC. Dissertação de Mestrado, Programa de Pós- Graduação da Universidade Comunitária Regional de Chapecó, SC, Brasil.

Gomes, A., Moretti, S. L. A. (2007). A responsabilidade e o social: uma discussão sobre o papel das empresas. São Paulo: Saraiva.

Greenfield, W. M. (2004). In the name of corporate social responsibility. Business Horizon, 47(1), 19-28.

Hair, J. F., Jr., Anderson, R. E., Tatham, R. L., Black, W. C. (2009). Análise multivariada de dados (6a ed.). Porto Alegre: Bookman.

Henseler, J., Ringle, C. M., Sinkovics, R. R. (2009). The use of partial least squares path modeling in international marketing. Advances in International Marketing, 20(1), 277-319.

Instituto Akatu. (2010). Responsabilidade social das empresas: percepção do consumidor brasileiro - pesquisa 2010. São Paulo: Autor. 
Irwin, J. R., Naylor, R. W. (2009). Ethical decisions and response mode compatibility: weighting of ethical attributes in consideration sets formed by excluding versus including product alternatives. Journal of Marketing Research, 46(2), 234-246.

Jacobi, P. (2006). Sustentabilidade ambiental, consumo e cidadania. Ambiente \& Sociedade, 9(1), 183-186.

Jöreskog, K., Sörbom, D. (2003). LISREL 8.54 student edition. Lincolnwood: Scientific Software International.

Kaiser, F. G. (1998) A general measure of ecological behavior. Journal of Applied Social Psychology, 28(5), 395-422.

Karna, J., Hansen, E., Juslin, H. (2003). Social responsibility in environmental marketing planning. European Journal of Marketing, 37(5/6), 848-871.

Karp, D. G. (1996). Values and their effect on pro-environmental behavior. Environment and Behavior, 28(1), 111-133.

Lages, N. S., Vargas, A., Neto. (2002). Mensurando a consciência ecológica do consumidor: um estudo realizado na cidade de Porto Alegre. Anais do Encontro da Associação Nacional de PósGraduação e Pesquisa em Administração, Salvador, BA, Brasil, 26.

Layrargues, P. P. (1998). A cortina de fumaça: o discurso empresarial verde e a ideologia da racionalidade econômica. São Paulo: Annablume.

Leite, A. P. R., Santos, T. C. (2007). Consumo consciente: uma análise na visão dos consumidores natalenses. Anais da Conferencia Regional de ISTR para América Latina y el Caribe, Salvador, BA, Brasil, 6.

Luchs, M., Naylor, R. W., Irwin, J. R., Raghunathan, R. (2010). The sustainability liability: potential negative effects of ethicality on product preference. Journal of Marketing, 74, 418-431.

Luthans, F., Youssef, C. M. (2007). Emerging positive organizational behavior. Journal of Management, 33(3), 321-349.

Maia, G. L., Vieira, E. G. D. (2004). Marketing verde: estratégias para produtos ambientalmente corretos. Revista de Administração Nobel, 31, 21-32.

Malheiros, M. A. P., Farhangmehr, M., Soares, A. M. S. (2010). Consumers' ethical behaviour: from what they think to what they do. Proceedings of the GIRA 2010 Conference on Corporate Governance, Innovation, Social and Environmental Responsibility, Lisbon, PT.

May, P., Lustosa, M. C., Vinha, V. (Orgs.). (2003). Economia do meio ambiente: teoria e prática. Rio de Janeiro: Elsevier.

McCormick, J. (1992). Rumo ao paraíso: a história do movimento ambientalista. Rio de Janeiro: Relume-Dumará.

Miles, M. P., Covin, J. G. (2000). Environmental marketing: a source of reputational, competitive and financial advantage. Journal of Business Ethics, 23(3), 299-311. 
Morh, L. A., Webb, D. J. (2005). The effects of corporate social responsibility and price on consumer responses. The Journal of Consumer Affairs, 39(1), 121-147.

Moretti, S. L. A. (2010). O marketing e o social em bancos brasileiros: evidências de relações assimétricas entre o discurso e a prática. Revista Brasileira de Marketing, 9(1), 124-150.

Moretti, S. L. A., Silva, D., Braga, S. S., Jr. (2010). Fatores de influência no consumo "verde": um estudo sobre o comportamento de compra no setor supermercadista. Anais do Encontro Nacional e I Encontro Internacional Sobre Gestão Empresarial e Meio Ambiente, Fortaleza, CE, Brasil, 12.

Motta, S. L. S., Rossi, G. B. (2001). A influência do fator ecológico na decisão de compra de bens de conveniência. Revista de Administração Mackenzie, 2(2), 109-130.

Moysés, J. E., Filho, Rodrigues, A. L., Moretti, S. L. A. (2009). Gestão social e ambiental em PMEs fornecedoras de grandes empresas: influência e poder sob a perspectiva da teoria dos stakeholders. Anais do Encontro Nacional e I Encontro Internacional Sobre Gestão Empresarial e Meio Ambiente, Fortaleza, CE, Brasil, 11.

Oliveira, B., Gouvêa, M., Guagliardi, J. (2004). A influência da responsabilidade social nas decisões de compra de produtos de conveniência. Anais do Encontro da Associação Nacional de Pós-Graduação e Pesquisa em Administração, Curitiba, PR, Brasil, 28.

Ottman, J. A. (1994). Marketing verde. São Paulo: Makron Books.

Parente, J. (2000). Varejo no Brasil: gestão e estratégia. São Paulo: Atlas.

Parente, J., Gelman, J. J. (Orgs.). (2006). Varejo e responsabilidade social. Porto Alegre: Bookman.

Pato, C. M. L., Tamayo, A. (2006). A escala de comportamento ecológico: desenvolvimento e validação de um instrumento de medida. Revista de Psicologia, 11(3), 289-296.

Porges, S. (2007). Ethical consumers and corporate responsibility: the market and trends for ethical products in food and beverage, personal care and household items. NewYork: Packaged Facts.

Porter, M. E., Kramer, M. R. (2005). A vantagem competitiva da filantropia corporativa. In M. V. Rodrigues y Rodrigues (Org.), Ética e responsabilidade social nas empresas (A. C. C. Serra, Trad.) (pp. 134-166). Rio de Janeiro: Elsevier.

Portilho, F. (2010). Sustentabilidade ambiental, consumo e cidadania (2a ed.).São Paulo: Cortez.

Pringle, H., Thompson, M. (1999). Brand spirit: how cause related marketing builds brands. Chichester: Wiley.

Puncheva, P. (2008). The role of corporate reputation in the stakeholder decision-making process. Business \& Society, 47(3), 272-290.

Queiroga, F., Gouveia, V. V., Coutinho, M. P. L., Vasconcelos, T. C., Jesus, G. R. (2005). Escala de comportamentos socialmente responsáveis do consumidor: estudo preliminar de evidência de validade. Psicologia em Estudo, 10(1), 143-149. 
Raposo, S. (n.d.). Consumo consciente: economizar água e energia e comprar produtos ecológicos são algumas das maneiras de contribuir com a preservação ambiental. Recuperado em 10 de janeiro, 2011, de http://www.salveoplaneta.com.br/art_01.htm.

Ringle, C.M., Wende, S., Will, A. (2005). SmartPLS 2.0 M3 (beta). Hamburg: University of Hamburg.

Roberts, J. A. (1996). Will the real socially responsible consumer please step forward? Business Horizons, 39, 79-83.

Sen, S., Bhattacharya, C. B. (2001). Does doing good always lead to doing better? Consumer reactions to corporate social responsibility. Journal of Marketing Research, 38, 225-243.

Seyfang, G., Paavola, J. (2008). Inequality and sustainable consumption: bridging the gaps. Local Environment, 13(8), 669-684.

Sheth, J., Parvatiyar, A. (1995). Ecological imperatives and the role of marketing. In M. Polonsky \& A Mintu-Winsatt (Orgs.), Environmental marketing: strategies, practice, theory and research (pp. 3-22). New York: The Haworth Press.

Simpson, I., Carmon, Z., Dhar, R., Drolet, A., Nowlis, S. M. (2001). Consumer research: in search of identity. Annual Review Psychology, 75, 52-249.

Sisodia, R., Sheth, J., Wolfe, D. B. (2007). Firms of endearment. Upper Saddle River, NJ: Wharton School Publishing.

Straughan, R. D., e Roberts, J. A. (1999). Environmental segmentation alternatives: a look at green consumer behavior in the new millennium. Journal of Consumer Marketing, 16(6), 558-575.

Tat Keh, H., Park, S. Y. (1997). To market, to market: the changing face of grocery retailing. Long Range Planning, 30(6), 836-846.

Urdan, A. T. (2001). Os consumidores recompensam o comportamento ético? Revista de Administração, 36(2), 6-15.

Vogel, D. (2005). The market for virtue - the potentials and limits of corporate social responsibility. Washington DC: Brooking Institution Press.

Wilder, A. (2003). Mudança no setor supermercadista e a formação de associações de pequenos supermercados. Dissertação de Mestrado, Escola Superior de Agricultura Luiz de Queiroz/USP, Piracicaba, SP, Basil..

Xueming, L., Bhattacharya, C. B . (2006). Corporate social responsibility, customer satisfaction, and market value. Journal of Marketing, 70(4), 1-18.

Data do recebimento do artigo: 08/07/2011

Data do aceite de publicação: 10/08/2012 